\title{
Model-based iterative reconstruction using higher-order regularization of dynamic synchrotron data
}

\author{
Daniil Kazantsev ${ }^{1,2}$, Enyu Guo ${ }^{1,2}$, A.B. Phillion ${ }^{3}$, Philip J. \\ Withers $^{1,2}$, Peter D. Lee ${ }^{1,2}$ \\ ${ }^{1}$ The Manchester X-Ray Imaging Facility, School of Materials, The University of \\ Manchester, Manchester, M13 9PL, UK \\ ${ }^{2}$ The Manchester X-Ray Imaging Facility, Research Complex at Harwell, Didcot, \\ Oxfordshire, OX11 0FA, UK \\ ${ }^{3}$ Department of Materials Science and Engineering, McMaster University, Hamilton, \\ L8S 4L7, Canada \\ E-mail: daniil.kazantsev@manchester.ac.uk
}




\begin{abstract}
We present a novel iterative reconstruction method applied to in situ Xray synchrotron tomographic data of dendrite formation during the solidification of magnesium alloy. Frequently, fast dynamic imaging projection data are undersampled, noisy, of poor contrast, and can contain various acquisition artifacts. Direct reconstruction methods are not suitable and iterative reconstruction techniques must be adapted to the existing data features. Normally, an accurate modelling of the objective function can guarantee a better reconstruction. In this work, we design a special cost function where the data fidelity term is based on the Group-Huber functional to minimize ring artifacts and the regularization term is a higher-order variational penalty. We show that the total variation penalty is unsuitable for some cases and higher-order regularization functionals can ensure a better fit to the expected properties of the data. Additionally, we highlight the importance of 3D regularization over $2 \mathrm{D}$ for the problematic data. The proposed method shows a promising performance dealing with angular undersampled noisy dynamic data with ring artifacts.
\end{abstract}

\title{
1. Introduction
}

Synchrotron X-ray microtomography $(\mu \mathrm{CT})$ is a powerful non-destructive modality which provides an opportunity to explore the inner structure of various materials in three dimensions (3D). Nowadays, many $\mu \mathrm{CT}$ experiments can be performed dynamically, so as to track the evolution of structure or microstructure as a function of time (4D imaging). Currently, synchrotron $\mu \mathrm{CT}$ imaging can provide fast exposure times and rotation speeds [1]-[4]. Although the acquisition speed is high, motion (an object's structural change during the time of a scan) is frequently present in the acquired data. Unfortunately, many in situ experiments are rapid and not easily controlled, for instance, imaging of geological processes [5, 6], semi-solid materials [7, 8], energy materials [9], biomaterials [10], or solidification experiments [11]-[16]. Therefore, in order to minimize motion artifacts, one might consider taking less projections or/and reducing exposure time. However, the former leads to angular undersampling and associated aliasing artifacts, while the later may lead to high noise (due to poorer photon statistics) and loss of contrast in the reconstructed images.

The tomographic acquisition process is based on collecting projections at multiple angles within $[0, \pi]$ angular range due to synchrotron $\mu \mathrm{CT}$ parallel beam geometry. Once the projections have been acquired, they are stacked into a sinogram from which the reconstructed image can be derived. When measurements are sampled densely and over a full angular range, it is possible to recover the attenuation coefficient distribution using computationally efficient direct reconstruction methods, such as Filtered Back Projection (FBP) or Fourier methods [17]. Frequently, direct reconstruction methods are not suitable for dynamic data since measurements are sparse, of low contrast, and noisy [6, 18]. Additionally, the $\mu \mathrm{CT}$ data can contain various acquisition inaccuracies or artifacts. Common artifacts include random errors in detector elements (zingers) as a result of particles scattering and consistent error-offsets in miscalibrated or defective 
detectors. In the reconstructed images, the former leads to streaks and the later to rings artifacts [19]-[21]. Both types of artifacts can cause significant difficulties when segmenting, quantifying, and post-processing the reconstructed data.

Iterative Image Reconstruction (IIR) techniques are better adapted to deal with noisy, angular undersampled, and erroneous measurements. IIR methods allow for more accurate modelling of the acquisition process and can incorporate different types of $a$ priori information (e.g. non-negativity, smoothness, sparsity) about the solution [22]. IIR approaches can be divided into deterministic and statistical or model-based methods 23. Deterministic methods are designed to solve a system of linear algebraic equations and normally rely on the assumption of Gaussian noise in the data. On the other hand, statistical approaches can incorporate more realistic noise models for low-dose or fast imaging. Therefore, statistical IIR methods can provide more stable solutions than deterministic techniques. Furthermore, model-based IIR methods can consider non-standard data fidelity terms to compensate for the variety of acquisition artifacts [19]-21].

Although the use of an accurate data model is highly important for the IIR algorithm, the choice of a regularizer can be also crucial. Nowadays, the Total Variation (TV) [24, 25] regularization penalty is widely used to achieve piecewise-constant $\ell_{1}$ type recovery. The gradient-based sparsifying characteristics of the TV penalty are remarkable; however, there are also some drawbacks associated with the penalty. Firstly, there is a loss of contrast, which to an extent, can be resolved using a Split-Bregman method for TV minimization problem [25]. Secondly, it is the cartoon or staircase appearance of the recovered images, i.e. all ramp-like features are lost. There have been various attempts to reduce the cartoon appearance by considering higher-order terms and various $\left(\ell_{1}+\ell_{2}\right)$-type combinations [26]-[31]. Recently, Chan et al. [32] compared various higher-order regularizers in one tomographic reconstruction framework. The authors concluded that the averaged combination of the TV penalty and higher-order (Laplacian) term can deliver better results than some state-of-the-art models, such as Total Generalized Variation (TGV) [29] or the Chan-Marquina-Mulet model [30]. In this work, we will use the $\left(\ell_{1}+\ell_{2}\right)$-type regularizer, but in a different form than has been used by Chan et al. [32].

Here we propose a data-tailored model-based IIR method which delivers easily segmentable reconstructions of high quality. Using in situ tomographic data of timeevolving dendritic structures, we investigate various properties of the method. Through the choice of different data terms and regularization penalties we demonstrate the importance of accurately defining the objective for IIR strategy. Although the method is novel in this form, it consists of previously thoroughly investigated elements that have been independently validated [21, 32]. In this work, we do not include synthetic phantom simulations but instead use real data to highlight important issues of modelbased IIR. We also provide open-source software which reproduces the results presented and can be used reconstructing similar datasets in future [33]. 


\section{Problem setting and reconstruction framework}

\subsection{Forward model formulation}

Image formation relies on the attenuation of X-rays and the mathematical model we adopt in this paper is based on three basic assumptions: beam monochromaticity, absence of diffraction or refraction, and Beer's law [17]:

$$
\bar{\Lambda}_{i}=I_{0, i} \exp \left(-\int_{L_{i}} \mu(\mathbf{r}) d l\right), i=1, \ldots, m,
$$

where $\mathbf{r} \in \mathbb{R}^{2}$ is the spatial position, $\mu$ is the attenuation coefficient of the object under investigation, $I_{0, i}$ is the intensity of the incoming beam, $\bar{\Lambda}_{i}$ is the intensity of the beam at the detector of the $i$-th ray integrated along the line path $L_{i}$, and $m$ is the total number of rays. Taking the negative log of the relative intensity leads to a linear relationship between the attenuation coefficient and the observations:

$$
\int_{L_{i}} \mu(\mathbf{r}) d l=-\ln \left(\frac{\bar{\Lambda}_{i}}{I_{0, i}}\right), i=1, \ldots, m .
$$

Representing the attenuation coefficient on a square grid of $n$ pixels: $\left\{\chi_{j}(\mathbf{r})\right\}_{i=1}^{n}$, i.e.,

$$
\mu(\mathbf{r})=\sum_{j=1}^{n} x_{j} \chi_{j}(\mathbf{r}),
$$

we obtain a discrete, linear, forward model:

$$
\mathbf{b}=\mathbf{A x},
$$

where $\mathbf{x} \in \mathbb{R}^{n}$ is a vector with attenuation coefficients $x_{j}, \mathbf{b} \in \mathbb{R}^{m}$ is a vector with the $\log$-corrected measurements, $b_{i}=-\ln \left(\frac{\bar{\Lambda}_{i}}{I_{0, i}}\right)$, and $\mathbf{A} \in \mathbb{R}^{m \times n}$ is the projection matrix with elements $a_{i j}=\int_{L_{i}} \chi_{j}(\mathbf{r}) d l$ which capture the contribution of the $i$-th ray to the $j$-th pixel.

\subsection{Objective function}

The measured transmission data (1) normally contain Possion noise due to the photoncounting process. We model the actual measured intensities $\Lambda_{i}$, as a Poisson random variable with parameter $\overline{\Lambda_{i}}[34]$ :

$$
\Lambda_{i} \sim \operatorname{Poiss}\left\{\bar{\Lambda}_{i}\right\}=\operatorname{Poiss}\left\{I_{0, i} e^{-[\mathrm{A} x]_{i}}\right\}, i=1, \ldots, m
$$

One can approach the reconstruction problem from the Bayesian perspective by employing the maximum a posteriori (MAP) estimate [19, 21, 34]:

$$
\underset{\mathbf{x}}{\arg \min } \sum_{i=1}^{m}\left[I_{0, i}[\mathrm{~A} \mathbf{x}]_{i}+\Lambda_{i} e^{-[\mathrm{A} \mathbf{x}]_{i}}\right]+\mathrm{L}_{\text {prior }}(\mathbf{x}),
$$

where $\mathrm{L}_{\text {prior }}$ is the negative log of the prior density function.

In [34, a quadratic approximation to the likelihood function (6) was proposed, leading to:

$$
\hat{\mathbf{x}}=\underset{\mathbf{x}}{\arg \min } \frac{1}{2}(\mathbf{A x}-\mathbf{b})^{\top} \boldsymbol{\Lambda}(\mathbf{A} \mathbf{x}-\mathbf{b})+\mathrm{L}_{\text {prior }}(\mathbf{x})
$$


where $\Lambda \in \mathbb{R}^{m \times m}$ is a diagonal matrix with entries $\left\{\Lambda_{i}\right\}_{i=1}^{m}$. The data term is the so-called Penalized Weighted Least Squares (PWLS) model, which is a simple approximation to the Poisson model (5) and is used for many X-ray tomography cases [19, 21, 34, 35]. The matrix $\boldsymbol{\Lambda}$ effectively gives a higher weight to projections with a higher photon-count. In the remainder of the paper we absorb these weights in the system of equations, i.e. redefining $\mathbf{A} \equiv \sqrt{\Lambda} \mathrm{A}$ and $\mathrm{b} \equiv \sqrt{\Lambda} \mathbf{b}$.

We now express the corresponding regularized optimization problem in the general form:

$$
\underset{\mathbf{x}}{\arg \min } f(\mathbf{A x}-\mathbf{b})+g(\mathbf{x})
$$

where $f: \mathbb{R}^{m} \rightarrow \mathbb{R}_{+}$is a data misfit term, $g: \mathbb{R}^{n} \rightarrow \mathbb{R}_{+}$is a convex regularization penalty. In the next sections we introduce the misfit data term $f$ and regularization term $g(\mathbf{x})$.

\subsection{Data misfit}

The choice of the data misfit term is driven by the nature of experimental data which contain multiple ring artifacts. It has been shown in [20, 21] that a specific form of the Huber misfit can be used to compensate for consistent offsets in data:

$$
f(\mathbf{r})=\min _{\mathbf{s}} \frac{1}{2}\left\|\mathbf{s}-\mathbf{B}^{\top} \mathbf{r}\right\|_{2}^{2}+\lambda\|\mathbf{s}\|_{1},
$$

where $\mathbf{B}=\left(\mathbf{I}_{m_{1}} \otimes \mathbf{1}_{m_{2}}\right) / \sqrt{m_{2}}$ with $\mathbf{B}^{\top} \mathbf{B}=\mathbf{I}_{m_{1}}$, here $m_{1,2}$ are the angular and the detector dimensions respectively. The matrix $\mathbf{B}$ sums the residuals in $m_{1}$ groups so that $\mathbf{s}$ is a vector of length $m_{2}$. The reasoning behind this formulation is that the outliers are likely to be correlated column-wise.

We now find the following closed-form solution of (9)

$$
\widehat{s}_{i}=\mathcal{S}_{\lambda}\left(\left(\mathbf{B}^{\top} \mathbf{r}\right)_{i}\right)
$$

where $\mathcal{S}_{\lambda}$ is the (element-wise) soft-thresholding operator given by

$$
\mathcal{S}_{\lambda}(r)=\left\{\begin{array}{rcc}
r+\lambda & \text { if } & r<-\lambda \\
0 & \text { if } & |r| \leq \lambda . \\
r-\lambda & \text { if } & r>\lambda,
\end{array}\right.
$$

Eq. 10 can be also written in terms of the conventional Huber misfit, one can find more details in [21]. Sparse minimization (9) enables the recognition of outliers in the 1D vector $\mathbf{B}^{\top} \mathbf{r}$. The vector of offsets is obtained to compensate for consistent intensity shifts in the sinogram domain. Notably, this model is valid only for stripes with a constant detector offset and will introduce bias for incomplete stripes and/or with variable offsets. As long as the majority of ring artifacts are the result of a constant detector offset, this approach remains consistent. 


\subsection{Higher-order regularization}

The choice of a regularizer in the objective function (8) is highly important in achieving realistic reconstructions. Taking into account the piecewise-smooth nature of the dendritic morphology of the case study being considered here (see section 3.1), we propose the following combined regularization term:

$$
g(\mathbf{x})=\beta_{1} g_{1}(\mathbf{x})+\beta_{2} g_{2}(\mathbf{x}),
$$

where the first-order isotropic TV-term [24, 25] is given as:

$$
g_{1}(\mathbf{x})=\left\|\mathrm{D}^{1} \mathbf{x}\right\|_{1}=\sqrt{\left[D_{1}^{1} \mathbf{x}\right]^{2}+\left[D_{2}^{1} \mathbf{x}\right]^{2}+\left[D_{3}^{1} \mathbf{x}\right]^{2}},
$$

and the second-order Lysaker, Lundervold, and Tai (LLT) term [26, 27]:

$$
g_{2}(\mathbf{x})=\left\|\mathrm{D}^{2} \mathbf{x}\right\|_{1}=\sqrt{\left[D_{1}^{2} \mathbf{x}\right]^{2}+\left[D_{2}^{2} \mathbf{x}\right]^{2}+\left[D_{3}^{2} \mathbf{x}\right]^{2}},
$$

where $D_{k}^{1,2}$ are discrete first and second derivatives in direction $k$, respectively. Note that the mixed derivative terms are dropped in (14) due to the simplicity of the formulation [26]. In section 3 we justify why formulation $(12)$ is more appropriate for the presented data. We present the whole algorithm based on proximal operators in the next section.

\subsection{Proximal operators framework and the reconstruction algorithm}

A generic way to solve problems of the form (8) is by a proximal-gradient method [36]:

$$
\mathbf{x}_{k+1}=\operatorname{prox}[g]\left(\mathbf{x}_{k}-L^{-1} \cdot \mathbf{A}^{\top} \nabla f\left(\mathbf{A x}_{k}-\mathbf{b}\right)\right),
$$

where $L$ is the Lipschitz constant of $\nabla f$ and the proximal step prox $[g]$ consists of two sub-problems using regularization terms (13 14):

$$
\begin{aligned}
& \operatorname{prox}_{\beta_{1}}\left[g_{1}\right](\mathbf{y})=\underset{\mathbf{x}}{\arg \min } g_{1}(\mathbf{x})+\frac{1}{2 \beta_{1}}\|\mathbf{x}-\mathbf{y}\|_{2}^{2}, \\
& \operatorname{prox}_{\beta_{2}}\left[g_{2}\right](\mathbf{y})=\underset{\mathbf{x}}{\arg \min } g_{2}(\mathbf{x})+\frac{1}{2 \beta_{2}}\|\mathbf{x}-\mathbf{y}\|_{2}^{2} .
\end{aligned}
$$

The obtained solutions of (15) and (16) can be equally averaged to obtain the approximated piecewise-smooth solution as it is shown in step 4 of Alg. 11. The similar proximal averaging strategies have been validated previously in [38, 39].

In Alg. 1 we present a detailed step-by-step approach for minimizing the objective (8). Let us consider all steps in details. Step 1 shows the accelerated PWLS problem solved for the data misfit in (7). The matrix G has been chosen as a high-pass 'sinc' filter in order to damp down the presence of low frequencies and accelerate convergence [23, 20, 35]. The Lipschitz constant $L$ has been automatically calculated using the Power method [37]. Step 2 involves of solving the minimization subproblem (15) with the TV penalty (13). Here we employ the Split-Bregman (SB) algorithm to solve the TV minimization problem exactly, without smoothing of the $\ell_{1}$ norm [25]. The SB algorithm is known to be fast in convergence and also helps reducing the loss of contrast in TViterations. For the data with such low signal-to-noise ratio (SNR) it is important to boost contrast while keeping the noise level as low as possible. The details of implementation 


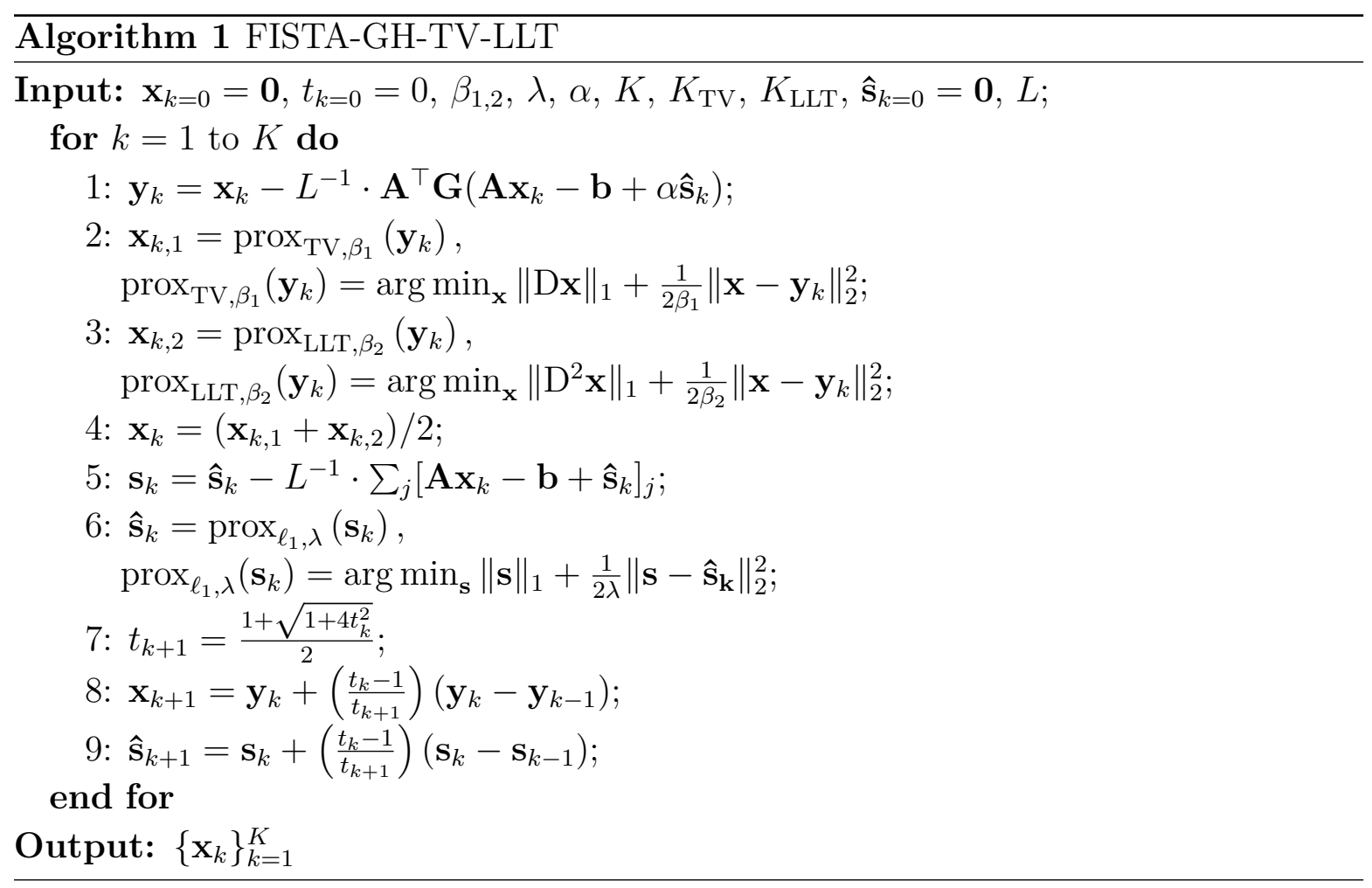

of the SB method are given in [25]. Step 3 involves finding the solution of another weighted denoising problem which consists of higher-order LLT term [27]. Optimization employing the LLT penalty requires solving the fourth-order partial differential equation (PDE). We use an explicit update scheme with a small tolerance parameter $\epsilon$ to avoid singularity [24, 26, 27]. In step 4, two solutions from steps 2 and 3 are averaged. The averaging operation provides an approximation to piecewise-smooth solution under appropriate choice of the regularization parameters $\beta_{1,2}$. Step 5 involves an update which corresponds to solving (9) for the data fidelity term. This step produces a 1D vector, which is the result of the sinogram residual summation over all angles. Step 6 involves of solving problem (9) for the $\ell_{1}$-term using the soft-thresholding operator (11). Steps (7-9) are the FISTA-related updates [37]. In the next section we present and comment on parameters of Alg. 1.

\section{Results}

\subsection{Tomographic data acquisition}

Solidification processes have been a focus of materials science for a long time [11][16. The formation of dendritic structures and their evolution during melting is of particular interest as these dendritic structures significantly affect the properties of the material. The dendrites usually exhibit a complex morphology in 3D, depending on various conditions including solute elements and cooling conditions, etc. Dendrite formation is critically dependent on the cooling, hence growth rate cannot be slowed 
down to meet the requirements of the image acquisition process without changing the nature of the process. Due to motion and the lack of temporal and spatial resolution involved at early stages of solidification, blurred and noisy surfaces can be reconstructed. This makes the detailed quantitative analysis of the dynamic changes in the dendrite evolution very difficult, or at best less accurate. Additionally, the surface of the dendrite is relatively smooth and therefore it is important to ensure a smooth recovery of the shapest.

The study was designed to perform X-ray imaging of an Mg-25wt.\%Zn alloy sample during solidification. The sample was heated to $30^{\circ} \mathrm{C}$ above the liquidus temperature of the alloy and then held at that temperature for 30 min ensuring complete melting of the sample. The sample was then cooled down at $3^{\circ} \mathrm{C} / \mathrm{min}$, allowing dendrites to form and develop. A relatively slow cooling rate was chosen to minimize possible motion-related blur in the reconstructed images induced by the fast dendritic growth.

Concurrent with the cooling of the sample, tomographic data acquisition was performed using a pink beam at the Diamond-Manchester Branchline (I13) of the Diamond Light Source. The apparatus and the set-up employed was very similar to [12. Projections were obtained using the high speed CCD camera (PCO Edge) and binned to $1280 \times 1080$ pixels. This resulted in a voxel size of $1.6 \mu \mathrm{m} \times 1.6 \mu \mathrm{m} \times$ $1.6 \mathrm{\mu m}$. One large tomographic dataset containing 82080 projections was collected at angular increments of $0.5^{\circ}$, and an exposure time of $20 \mathrm{~ms}$ per projection. The sample was rotated continuously in one direction. Over $180^{\circ}$ rotation a total of 360 projections were collected during 7.2s time interval; thus in total, 228 time-frames (volumes) were obtained during the acquisition experiment. The short exposure time and small number of projections per rotation relative to the detector size make reconstruction of this data a challenging task.

\subsection{Direct reconstruction}

Before assessing the reconstructions using Alg. 1, we reconstructed the data using the FBP algorithm with the 'Ram-Lak' filter [17]. Fig. 11 shows an example of 2D slice from the reconstructed $1 \mathrm{k}^{3}$ voxels volume extracted from the 140 th time-frame. At this time-point in the experiment, the dendritic structures are large and the growth speed is reduced substantially as compared to the initial time-frames. Correspondingly, the number of motion artifacts is reduced as compared to the initial time-frames. FBP reconstruction in 1 contains a significant amount of noise, has a poor contrast between the background and the dendrites, and multiple ring artifacts of different intensity and thickness. This image quality is typical of FBP-reconstructed images.

$\ddagger$ The smooth surfaces of dendrites can be seen in the scanning electron microscope (SEM) image in Fig. 11 of paper [40] 


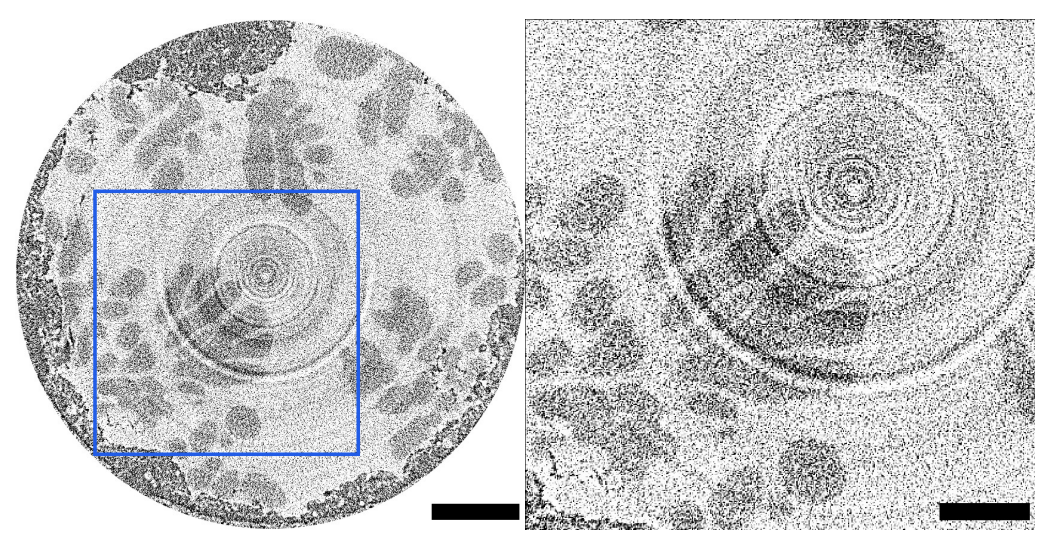

(a) FBP reconstruction

(b) magnified region

Figure 1: The reconstructed slice (a) using the FBP algorithm [17]. The scale bar (bottom right corner) corresponds to $300 \mu \mathrm{m}$; (b) the zoomed region. The scale bar corresponds to $100 \mu \mathrm{m}$. Note the significant amount of noise, low resolution, poor contrast, and highly prominent ring artifacts.

\subsection{Algorithm parameters and the effect of dimensionality on the reconstruction}

Unlike the FBP method, the proposed model-based iterative reconstruction method is able to create high quality $3 \mathrm{D}$ images. In this section we demonstrate how the dimensionality of the regularizer (2D and 3D) can affect the quality of the reconstructed image. It is known that by adding supplementary information into a regularizer one can significantly improve signal-to-noise ratio of reconstructed images [6, 18, 19]. Here we demonstrate how important this can be for problematic real data cases.

Synchrotron $\mu \mathrm{CT}$ data imaging employs the parallel-beam scanning geometry which largely simplifies big data handling since each slice can be reconstructed independently [41. The whole volume can be reconstructed in a slice-by-slice manner considering each slice serially on a PC, or in parallel across a cluster. Therefore, slice-byslice reconstruction can be efficiently realized with a minimal memory and processor requirements. It is also suitable for highly efficient GPU-processing, even for big datasets the $3 \mathrm{D}$ reconstruction problem can be decomposed into series of $2 \mathrm{D}$ operations. Parallel beam iterative reconstruction normally consists of multiple applications of $2 \mathrm{D}$ forward and backward projection operators and also regularization operations which can be performed in 2D or 3D. Therefore the main difference between 2D and 3D IIR method for parallel beam geometry is the dimensionality of a regularizer. For 2D IIR case, terms $D_{3}^{1} \mathbf{x}$ and $D_{3}^{2} \mathbf{x}$ are absent in (13) and (14), respectively. For our experiments, we used on-the-fly forward and backward projection operations of the GPU accelerated modules from the ASTRA toolbox [42].

Now we present all reconstruction parameters of Alg. 11. The total number of outer FISTA iterations is set as $K=65$; the preconditioner $\mathbf{G}$ is selected as the 'sinc' function [17] to damp low frequencies in the sinogram residual [23, 20]. Parameter 


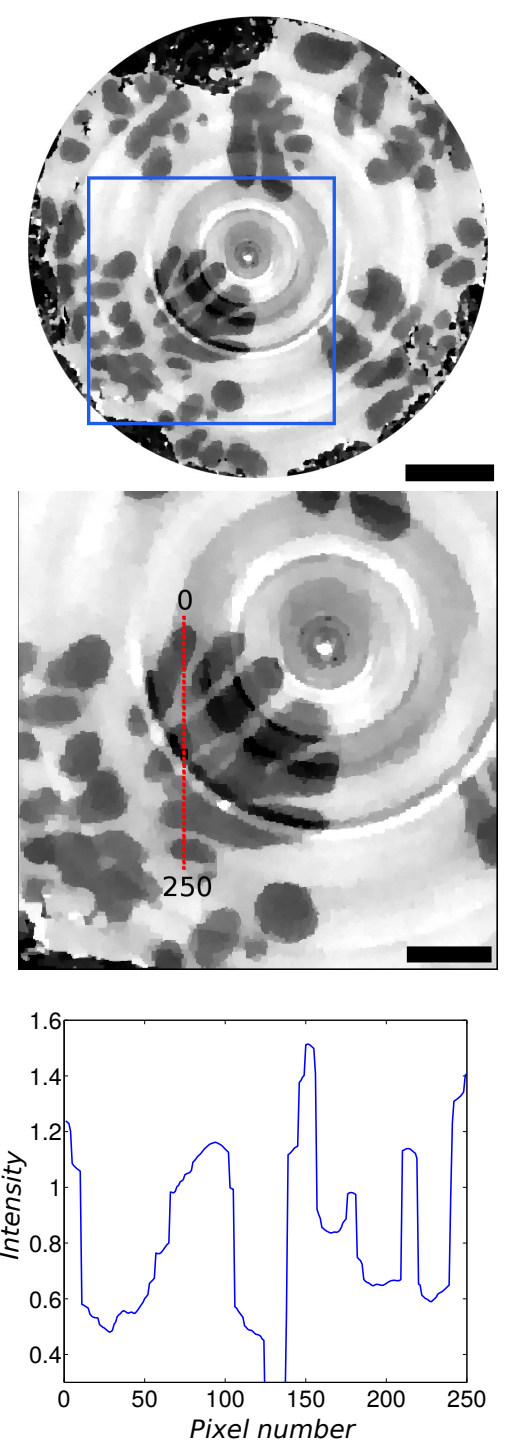

(a) LS-2DTV
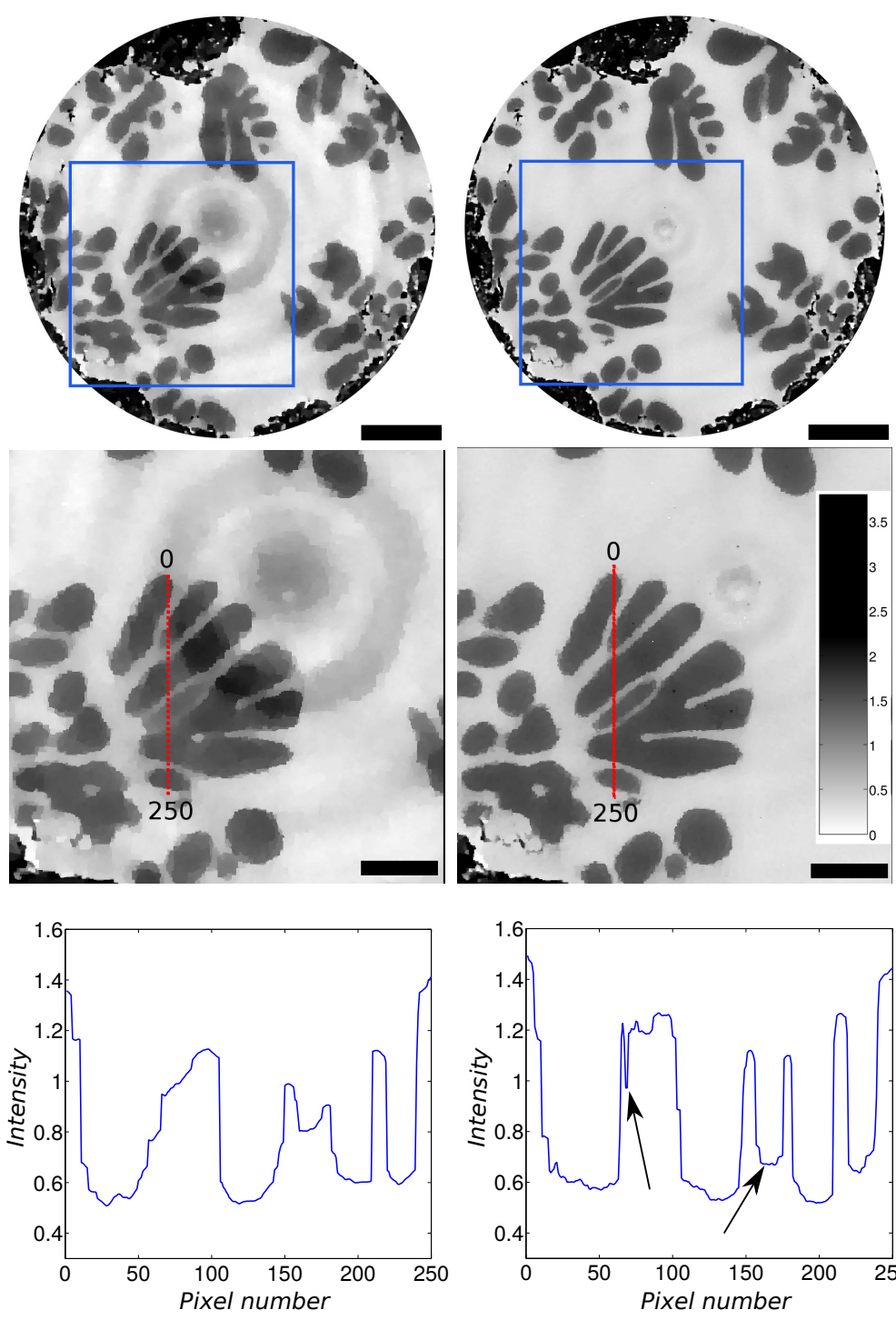

(b) GH-2DTV

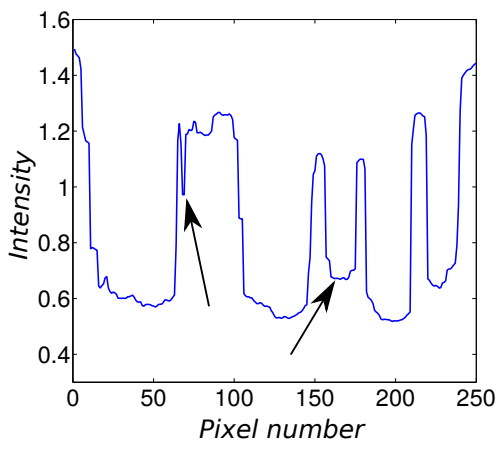

(c) GH-3DTV

Figure 2: The reconstructions of a slice from the volume using different methods: LS2DTV (a), GH-2DTV (b), and GH-3DTV (c). Top row: full-size reconstructed images. The scale bar corresponds to $300 \mu \mathrm{m}$. Middle: the magnified region with the selected profile for $1 \mathrm{D}$ plot. The scale bar corresponds to $100 \mu \mathrm{m}$. Bottom: 1D plots. Note the improved resolution, contrast, and the absence of rings for the GH-3DTV method compared to GH-2DTV, which is a 2D version of the same method. On the 1D plot for the GH-3DTV methods, arrows indicate regions which have been better resolved in terms of the resolution.

$\alpha=20$ (step 1) is introduced to accelerate convergence of the algorithm with respect to the ring removal process. The weighted vector of rings variables $\hat{\mathbf{s}}_{k}$ ensures a faster suppression of rings in iterations. Regularization parameters $\beta_{1}=100, \beta_{2}=250$ have been chosen accurately using the visual assessment of reconstructed images in terms of resolution versus noise and artifacts. Parameter $\lambda=0.002$ has been chosen to sparsify 
$1 \mathrm{D}$ vector of rings offsets $\hat{\mathbf{s}}_{k}$. In our computer implementation [33], we visualize the vector of rings offsets and identify parameter $\lambda$ according to the sparse representation of it (outliers can be detected visually). The maximum number of iterations for inner proximal problems have been chosen to $K_{\mathrm{TV}}=25$ and $K_{\mathrm{LLT}}=70$. However, inner iterations can be terminated earlier when the residual becomes small $\left\|\mathbf{x}_{k+1}-\mathbf{x}_{k}\right\|_{2}^{2} \leq \epsilon$, where $\epsilon=1 \times 10^{-4}$. The presented results can be reproduced using open-source codes in Matlab and $\mathrm{C}$ [33].

In Fig. 2 we show reconstructions using different methods. Note that for this experiment we do not use the LLT regularization scheme and therefore steps 3-4 have been excluded from Alg. 1. The LS-2DTV method can be derived from Alg. 1 when the ring variable equal to zero, i.e. $\alpha=0$ and steps 5,6 , and 9 are excluded. The GH-2DTV and GH-3DTV methods are with 2D TV and 3D TV regularization, respectively.

The reconstructed images in Fig. 2 demonstrate that without modelling of the rings variable, the LS-2DTV method delivers reconstructions with strong ring artifacts. The GH-2DTV method removes sharp ring artifacts, however the shadows of some rings remain. Also the resolution of $2 \mathrm{D}$ reconstructions is not satisfactory and some features have been blurred. In the profiles, see the area in the middle ( $x$-axis 150-200), dendritic arms have merged. The reconstruction using the GH-3DTV method delivers much better quality in terms of resolution, contrast, and the absence of ring artifacts. Notably the 1D profile of GH-3DTV confirms that the contrast has been improved significantly, the arrows indicate features which have been improved by the 3D version of the method. One can conclude that in some cases $2 \mathrm{D}$ regularization is not sufficient to ensure high-quality reconstruction and 3D regularization should be used instead.

In the next section we will study how the nature of the chosen regularizer can affect reconstruction and segmentation.

\subsection{The effect of a higher-order regularizer on reconstruction}

Higher-order regularization can produce more realistic images due to preservation of the ramp-like features. Although the TV regularization can produce images of high contrast with very distinct and sharp edges (see Fig. 2) it can be unsuitable when applied to data with smoother features. The surfaces of dendritic arms in metals are known to be smooth [11]-[16], [40], therefore the assumption of piecewise-constant recovery using the TV term does not hold. In order to achieve a piecewise-smooth solution, we added an additional higher-order regularization term and the resulting solution is averaged with the TV term (see details in Section 2.4). Previously, in Section 3.3, we have shown that the use of 3D regularization is crucial for some reconstruction tasks, therefore for our experiments we will use 3D regularization. We will remove the '3D' abbreviation from the subsequent notations.

In Fig 3, we demonstrate reconstruction using two methods: GH-TV and GH-TVLLT. The latter method is exactly Alg. 1 and the former is without the LLT-related steps, i.e. $3-4$. 

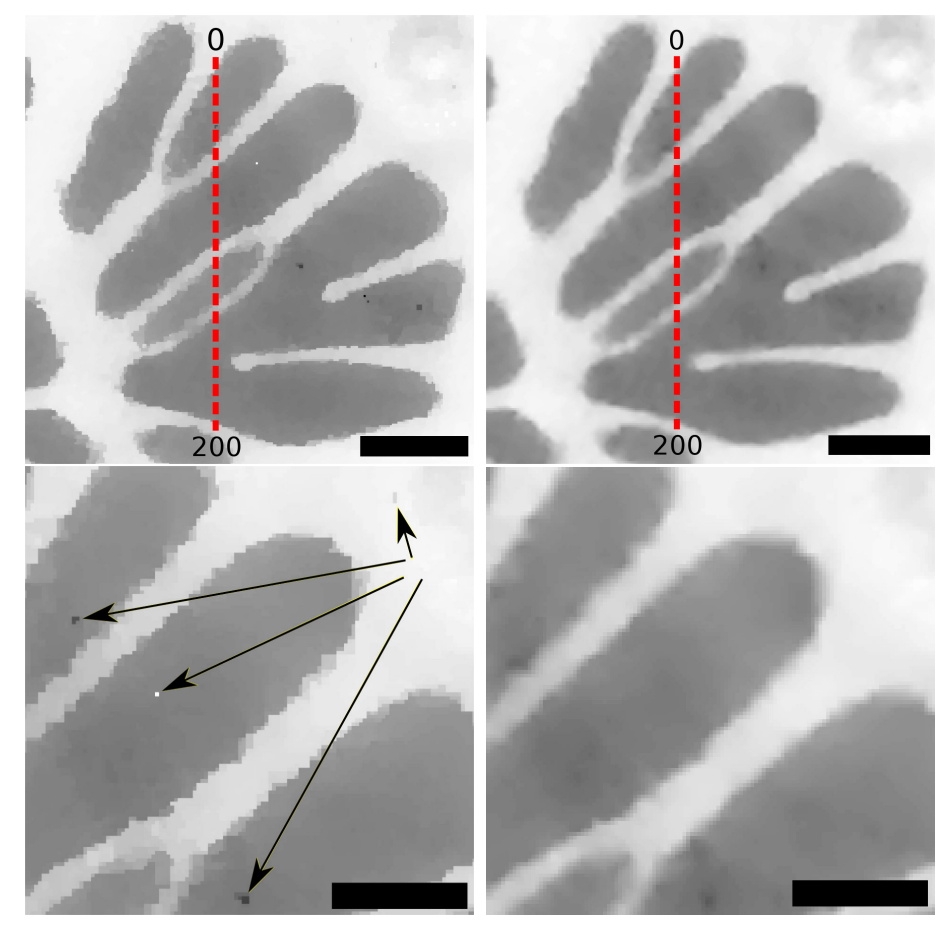

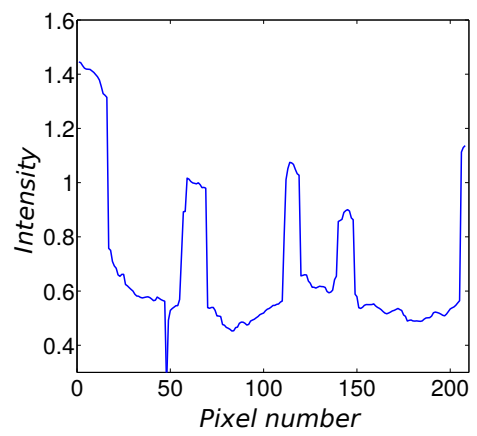

(a) GH-TV

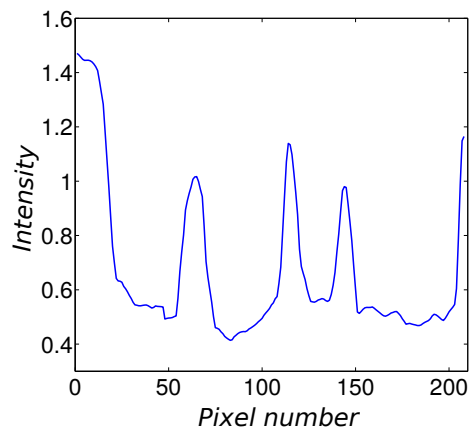

(b) GH-TV-LLT

Figure 3: The reconstruction using the GH-TV method (a) and the GH-TV-LLT method (b). Top row: the reconstructed dendritic structure with 1D vertical profile crossing several branches. The scale bar corresponds to $50 \mu \mathrm{m}$. Middle: the magnified region shows the surfaces of dendritic arms more clearly. Notice the ragged edges of dendritic branches, blocky background, and some outliers (indicated by arrows) with the GH-TV reconstruction. The scale bar corresponds to $25 \mu \mathrm{m}$. Bottom: 1D profiles across the dendritic branches. The GH-TV-LLT profile shows much smoother shapes of dendrites and also the individual arms have been resolved better than with the GH-TV method.

In the reconstructed images one can see that the GH-TV reconstruction is very blocky yet appears to be slightly sharper than the recovery with the GH-TV-LLT method. With the GH-TV recovery, the dendritic branches have saw-shaped surfaces and also a few isolated outliers of different intensities (indicated by arrows). These clusters of outliers can be explained by the nature of the TV penalty to preserve abrupt intensity variations as step-functions, leading to accentuation of some undesirable 
features (apparently noise in this case). The $1 \mathrm{D}$ profile through one of outliers also shows a distinctive dip of intensity around the value of 50 of the $x$-axis. The shapes of features with the GH-TV method are not smooth but rugged due to effects of the TV penalty. The reconstruction using the GH-TV-LLT method might not look as sharp as with the GH-TV method, however the surfaces of branches are recovered much smoother. Additionally in the zoomed image, the absence of clustered outliers is evident. The 1D profile shows smooth local variations of intensity without significant jumps. Although the contrast (between the background and dendritic features) might seem visually lower for the GH-TV-LLT method, the 1D plot proves the opposite. The main features are well preserved and appear to be not oversmoothed. Furthermore, some features are resolved even better compared to the GH-TV case (see two peaks between 100 and 150 of the $x$-axis of $1 \mathrm{D}$ plots). Improved contrast of the GH-TV-LLT method is also confirmed by the segmentation results (see Fig. 4, top).

We used the automatic Otsu segmentation method [43] for both reconstructed volumes. The results of segmentation using GH-TV reconstructions provide partially

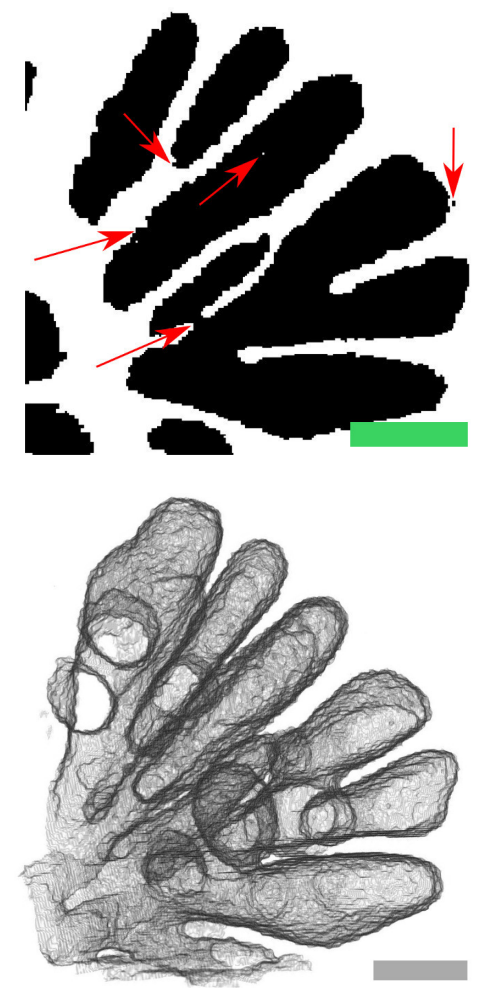

(a) GH-TV
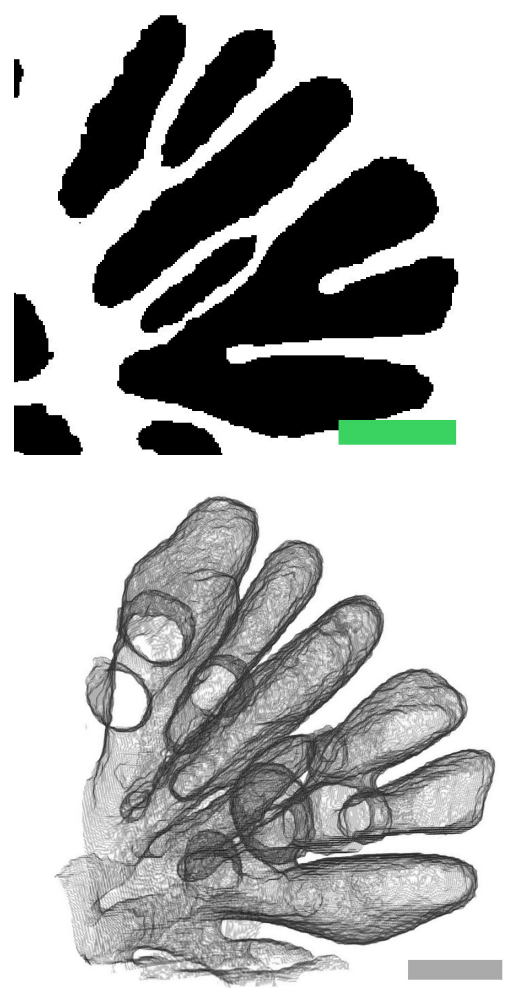

(b) GH-TV-LLT

Figure 4: Top row: the segmented reconstructed images for the GH-TV (a) and the GH-TV-LLT (b) methods using the Otsu method. The scale bar corresponds to $50 \mu \mathrm{m}$. Bottom: the rendered volumes using Drishti visualization software [44]. One can notice much smoother and accurate segmentation of the data reconstructed using the GH-TVLLT method (b). The scale bar corresponds to $50 \mu \mathrm{m}$. 
arrows). The segmented GH-TV-LLT data give smoother and more realistic contours of dendrites. Notably, the merging of dendritic arms can also be avoided by using the GH-TV-LLT reconstruction method. In Fig. 4 (bottom) we also provide a part of rendered segmented volume which corresponds to the same dendrite shown in images above. The rendering performed without smoothing in Drishti visualization software [44 and the resulted 3D volumes demonstrate rough surfaces with the GH-TV recovery, while smoother surfaces with the GH-TV-LLT method.

\section{Discussion and Conclusions}

In this manuscript, we have demonstrated a new iterative reconstruction algorithm capable of reconstucting $4 \mathrm{D}$ synchrotron $\mu \mathrm{CT}$ data of poor quality with minimal noise and high fidelity. We highlighted the importance of an accurate modelling of the objective function in iterative algorithm when applied to real data. We choose the case of time-evolving dendritic structures since it is an important topic researched by many scientists and encouraged by advances in synchrotron high-speed imaging. Similar experiments will continue to be challenging in terms of reconstruction from inaccurate and noisy data; therefore, one needs robust reconstruction algorithms to deal with various issues.

Although the presented GH-TV-LLT algorithm looks slightly cumbersome (see Alg. 11) it is easy to implement and the choice of parameters is straightforward (many are fixed for different applications). The main difficulty is the trade-off between $\beta_{1}$ and $\beta_{2}$ regularization parameters. We found that the quickest way to establish these parameters is to find $\beta_{1}$ first while keeping $\beta_{2}=0$. Once the $\beta_{1}$ parameter is established one can fix it and initiate the search over $\beta_{2}$ values. When selecting parameters, one should establish a trade-off between the level of smoothing and noise preservation. We observed that minor oversmoothing removes ring artifacts even better, while compromising the spatial resolution. When the fine resolution is not an issue, it is recommended to oversmooth slightly.

Another substantial problem is the time of calculation and memory resources required for $3 \mathrm{D}$ regularization of big data. In order to reconstruct $1 \mathrm{k}^{3}$ volume with Alg. 1 it can take approximately 15-20 hours using the following hardware: GeForce GTX 980 graphics card to perform 2D projection-backprojection on the GPU, CPU with 24 threads to calculate regularization and approximately 30GB of RAM. A significant amount of time $(\approx>85 \%)$ goes to the regularization routine running on the CPU. One option to accelerate the process would be performing regularization on subvolumes using a GPU or a cluster of GPUs. We are currently looking into the possibility of implementing this to make the present algorithm more practical to deal with large $4 \mathrm{D}$ datasets. Considering faster converging methods may also reduce the number of iterations substantially.

The proposed model-based iterative reconstruction technique consists of advanced elements: unconventional data fidelity term and higher-order regularization. By using 
the Group-Huber data functional, one can alleviate the majority of strong ring artifacts. The remaining artifacts can be further suppressed by using 3D regularization penalty which also boosts both image resolution and image contrast. It has been shown that the use of the correct model for regularization is highly important. Higher-order regularization provides more realistic images and more accurate segmentation when applied to real data of fast dendritic growth. The method provides an opportunity to reconstruct severely undersampled and noisy $4 \mathrm{D}$ data in high resolution which can be easily segmented.

\section{Acknowledgments}

This work was made possible by the facilities and support provided by the Research Complex at Harwell, funded in part by the EPSRC (EP/I02249X/1), as well as the CCPi initiative (EP/M022498/1). Some data used during this research and associated code are openly available at CCPForge [33. We acknowledge the facility use of Diamond Light Source (MT13302-1) and DLS staff for technical assistance.

\section{References}

[1] Drakopoulos M, Connolley T, Reinhard C, Atwood R, Magdysyuk O, Vo N, Hart M, Connor L, Humphreys B, Howell G and Davies S 2015 I12: the joint engineering, environment and processing (JEEP) beamline at diamond light source Journal of synchrotron radiation 22(3) $828-838$

[2] Mokso R, Marone F and Stampanoni M 2010 Real time tomography at the Swiss Light Source In AIP Conference Proceedings 1234(1) 87-90

[3] Maire E and Withers P J 2014 Quantitative X-ray tomography International materials reviews 59(1) $1-43$

[4] Baruchel J and Buffiere J Y, Cloetens P, Di Michiel M, Ferrie E, Ludwig W, Maire E and Salvo L 2006 Advances in synchrotron radiation microtomography Scripta Materialia 55(1) 41-6

[5] Pankhurst M J, Dobson K J, Morgan D J, Loughlin S C, Thordarson T, Lee P D and Courtois L 2014 Monitoring the magmas fuelling volcanic eruptions in near-real-time using X-ray microcomputed tomography Journal of Petrology 55(3) 671-84

[6] Kazantsev D, Thompson W M, Lionheart W R, Van Eyndhoven G, Kaestner A P, Dobson K J, Withers P J and Lee P D 2015 4D-CT reconstruction with unified spatial-temporal patch-based regularization Inverse problems and imaging $\mathbf{9 ( 2 )} 447-67$

[7] Kareh K M, Lee P D, Atwood R C, Connolley T and Gourlay C M 2014 Revealing the micromechanisms behind semi-solid metal deformation with time-resolved X-ray tomography Nature communications 54464

[8] Karagadde S, Lee P D, Cai B, Fife J L, Azeem M A, Kareh K M, Puncreobutr C, Tsivoulas D, Connolley T and Atwood R C 2014 Transgranular liquation cracking of grains in the semi-solid state Nature communications 68300

[9] Eastwood D S, Bayley P M, Chang H J, Taiwo O O, Vila-Comamala J, Brett D J, Rau C, Withers P J, Shearing P R, Grey C P and Lee P D 2015 Three-dimensional characterization of electrodeposited lithium microstructures using synchrotron X-ray phase contrast imaging Chemical Communications 51(2) 266-268

[10] Poologasundarampillai G, Yu B, Tsigkou O, Valliant E, Yue S, Lee P D, Hamilton R W, Stevens M M, Kasuga T and Jones J R 2012 Bioactive silica-poly ( $\gamma$-glutamic acid) hybrids for bone 
regeneration: effect of covalent coupling on dissolution and mechanical properties and fabrication of porous scaffolds Soft Matter 8(17) 4822-4832

[11] Guo E, Phillion A B, Cai B, Shuai S, Kazantsev D, Jing T and Lee P D 2017 Dendritic evolution during coarsening of Mg-Zn alloys via 4D synchrotron tomography Acta Materialia 123 373-82

[12] Shuai S, Guo E, Phillion A B, Callaghan M D, Jing T and Lee P D 2016 Fast synchrotron X-ray tomographic quantification of dendrite evolution during the solidification of $\mathrm{Mg}$-Sn alloys Acta Materialia 118 260-269

[13] Azeem M A, Lee P D, Phillion A B, Karagadde S, Rockett P, Atwood R C, Courtois L, Rahman K M and Dye D 2017 Revealing dendritic pattern formation in Ni, Fe and Co alloys using synchrotron tomography Acta Materialia 128 241-248

[14] Cai B, Karagadde S, Yuan L, Marrow T J, Connolley T and Lee P D 2014 In situ synchrotron tomographic quantification of granular and intragranular deformation during semisolid compression of an equiaxed dendritic Al-Cu alloy Acta Materialia 76 371-80

[15] Daudin R, Terzi S, Lhuissier P, Tamayo J, Scheel M, Babu N H, Eskin D G and Salvo L 2017 Particle-induced morphological modification of $\mathrm{Al}$ alloy equiaxed dendrites revealed by subsecond in situ microtomography Acta Materialia 125 303-10

[16] Gibbs J W, Mohan K A, Gulsoy E B, Shahani A J, Xiao X, Bouman C A, De Graef M and Voorhees P W 2015 The three-dimensional morphology of growing dendrites Scientific reports 511824

[17] Kak A C and Slaney M 1988 Principles of computerized tomographic imaging (New York: IEEE Press)

[18] Kazantsev D, Guo E, Kaestner A, Lionheart W R, Bent J, Withers P J and Lee P D 2016 Temporal sparsity exploiting nonlocal regularization for $4 \mathrm{D}$ computed tomography reconstruction Journal of X-ray science and technology 24(2) 207-19

[19] Mohan K A, Venkatakrishnan S V, Gibbs J W, Gulsoy E B, Xiao X, De Graef M, Voorhees P W and Bouman C A 2015 TIMBIR: A method for time-space reconstruction from interlaced views IEEE Transactions on Computational Imaging 1(2) 96-111

[20] Paleo P and Mirone A 2015 Ring artifacts correction in compressed sensing tomographic reconstruction Journal of synchrotron radiation, 22(5) 1268-1278

[21] Kazantsev D, Bleichrodt F, van Leeuwen T, Kaestner A, Withers P J, Batenburg K J and Lee P D 2017 A novel tomographic reconstruction method based on the robust Student's $\mathrm{t}$ function for suppressing data outliers IEEE Transactions on Computational Imaging doi: 10.1109/TCI.2017.2694607

[22] Vogel C R 2002 Computational methods for inverse problems 23 (SIAM)

[23] Bertero M and Boccacci P 1998 Introduction to inverse problems in imaging (CRC press)

[24] Rudin L I, Osher S and Fatemi E 1992 Nonlinear total variation based noise removal algorithms Physica D. 60 259-68

[25] Goldstein T and Osher S 2009 The split Bregman method for L1-regularized problems SIAM journal on imaging sciences $\mathbf{2 ( 2 )} 323-343$

[26] Lysaker M, Lundervold A and Tai X C 2003 Noise removal using fourth-order partial differential equation with applications to MRI in space and time IEEE Transactions on image processing 12(12) $1579-90$

[27] Lysaker M and Tai X C 2006 Iterative image restoration combining total variation minimization and a second-order functional International journal of computer vision 66(1) 5-18

[28] Papafitsoros K and Schönlieb C B 2014 A combined first and second order variational approach for image reconstruction Journal of mathematical imaging and vision 48(2) 308-338.

[29] Bredies K, Kunisch K and Pock T 2010 Total generalized variation SIAM Journal on Imaging Sciences 3(3) 492-526

[30] Chan T, Marquina A and Mulet P 2000 High-order total variation-based image restoration SIAM Journal on Scientific Computing 22(2) 503-16

[31] Liu Y, Liang Z, Ma J, Lu H, Wang K, Zhang H, and Moore W 2014 Total variation-stokes strategy 
for sparse-view X-ray CT image reconstruction IEEE transactions on medical imaging, 33(3) $749-763$

[32] Chan R H, Liang H, Wei S, Nikolova M and Tai X C 2015 High-order total variation regularization approach for axially symmetric object tomography from a single radiograph Inverse Problems and Imaging 9(1) 55-77

[33] Kazantsev D 2017 Matlab-C codes for FISTA-GH-TV-LLT reconstruction https://ccpforge. cse.rl.ac.uk/gf/project/ccpi_itr/frs/?action=FrsReleaseView\&release_id=555

[34] Sauer K and Bouman C 1993 A local update strategy for iterative reconstruction from projections IEEE Transactions on Signal Processing, 41(2) 534-48

[35] Ueda R, Yamazaki F and Kudo H 2017 FBP embedded iterative method to efficiently solve the Low-dose CT In SPIE Medical Imaging 1013234-1013234

[36] Parikh N and Boyd S 2014 Proximal algorithms Foundations and Trends in Optimization 1(3) $127-239$

[37] Beck A and Teboulle M 2009 A fast iterative shrinkage-thresholding algorithm for linear inverse problems SIAM journal on imaging sciences 2(1) 183-202

[38] Yu Y L 2013 Better approximation and faster algorithm using the proximal average In Advances in neural information processing systems 458-466

[39] Xu Q, Yang D, Tan J, Sawatzky A and Anastasio M A 2016 Accelerated fast iterative shrinkage thresholding algorithms for sparsity-regularized cone-beam CT image reconstruction Medical physics 43(4) 1849-72

[40] Chen R, Xu Q and Liu B 2015 Cellular automaton simulation of three-dimensional dendrite growth in Al-7Si-Mg ternary aluminum alloys Computational Materials Science 105, 90-100

[41] Wadeson N and Basham M 2016 Savu: A Python-based, MPI Framework for Simultaneous Processing of Multiple, N-dimensional, Large Tomography Datasets arXiv preprint arXiv:1610.08015

[42] Palenstijn W J, Batenburg K J and Sijbers J 2011 Performance improvements for iterative electron tomography reconstruction using graphics processing units (GPUs) J. of Struct. Biol. 176(2) $250-253$

[43] Otsu N 1975 A threshold selection method from gray-level histograms Automatica 11(285-296) $23-27$

[44] Limaye A 2012 Drishti: a volume exploration and presentation tool In Proc SPIE 8506 85060X 